\title{
A rare case of cardiac paraganglioma with mitral valve replacement is
}

\author{
Jignesh Kothari ${ }^{\text {a,c }}$, Parth Solanki ${ }^{\text {a,c, },}$, Ketav Lakhia ${ }^{\text {a,c }}$, Hiren Boraniya ${ }^{\text {a,c }}$, Himani Pandya ${ }^{\text {b,c }}$ \\ a Department of Cardiovascular and Thoracic Surgery \\ b Department of Research \\ c U.N. Mehta Institute of Cardiology and Research Center, Medical College, Civil hospital Campus, Asarwa, Ahmedabad 380016, India
}

\section{A R T I C L E I N F O}

\section{Article history:}

Received 31 January 2017

Received in revised form 27 March 2017

Accepted 8 May 2017

Available online 9 May 2017

\section{Introduction}

Paraganglioma is a tumor arising from the extra-adrenal paraganglion system. It is a type of neurogenic tumour commonly occurring in posterior mediastinum. One of the most unusual benign tumours affecting the heart is an intrapericardial paraganglioma. Paragangliomas are rare neoplasms of neural crest origin arising in the chromaffin (pheochromocytoma) and chemoreceptor (chemodectoma) tissues. Most of the paragangliomas are located in, or adjacent to, the left atrium. Patients with cardiac paragangliomas may present with arterial hypertension. Majority of paragangliomas appear to be sporadic. However, approximately one-third are associated with hereditary disorders. Some hereditary paragangliomas, particularly those arising in the head and neck, have been linked to mutations in the genes encoding different subunits of the succinate dehydrogenase (SDH) enzyme complex. Paraganglioma commonly occur in multiple endocrine neoplasia types $2 \mathrm{~A}$ and $2 \mathrm{~B}$ (MEN2), neurofibromatosis type 1 (NF1), von Hippel Lindau (VHL).

"Here we are presenting a case of an intrapericardial paraganglioma incidentally discovered in a 50 year-old male patient during preoperative work up for mitral valve replacement".

\section{Case details}

50 year old male presented with chief complain of dyspnea on exertion. At his physical examination, heart \& lung auscultation were suggestive of pansystolic murmur in mitral area radiated to the axilla. A 12 lead electrocardiography (ECG) was suggestive of left ventricular dilation and left atrial enlargement (biphasic p wave in lead V1 and bifid p wave in

\footnotetext{
th Peer review under responsibility of The Society of Cardiovascular Academy.

* Corresponding author at: U. N. Mehta Institute of Cardiology and Research Center, Medical College, New Civil Hospital Campus, Asarwa, Ahmedabad 380016, India.

E-mail address: parthdoc@yahoo.co.in (P. Solanki).
}

lead 2- p mitral with prominent negative wave). Lateral and postero anterior X-ray showed left atrial enlargement and cardiomegaly. Echocardiography showed moderate mitral stenosis with severe mitral regurgitation. As a part of the investigation protocol as the age of the patient was 50 years we performed conventional coronary angiography, in which we accidently found a mediastinal mass having vascular supply from right coronary artery and left circumflex artery. For further evaluation ECG gated cardiac contrast enhanced computed tomography was planned in which we came to know of a hyper enhancing mass lesion in middle mediastinum between left pulmonary artery and left atrium of size $7.4 \times 6.3 \times 5.4 \mathrm{~cm}$ most possibly a paraganglioma (Fig. 1 ). Before planning of surgery P adrenergic blockade for more than 1 week was done to prevent hypertensive crisis in case of accidental hyper secretion of catecholamine during dissection of the tumor during surgery. We performed mitral valve replacement along with mediastinal mass excision. After pericardiotomy mass was visible in transverse sinus between left pulmonary artery and left atrial appendage with dense adhesion present between mass and left pulmonary artery and left atrium. On further examination mass was found to be extending in the transverse sinus between aorta and main pulmonary artery and reaching superior vena cava with dense adhesion present in between (Fig. 2). So we went on cardio pulmonary bypass and did excision of the mass. After going on normothermic aorto bicaval bypass proper plane was found between the mass and adjacent cardiac structures and by meticulous dissection, the mass was completely removed along with the overlying capsule (Fig. 2). This mass excision was carried out without hypothermia. Due to severe adhesions with left atrium, the left atrial roof was repaired. After removal of the mass the heart was arrested with moderate hypothermic cardioplegia and mitral valve replacement was accomplished. After coming off cardio pulmonary bypass and decannulation in view of diffuse oozing all over the dissected areas and chest was left open. Patient was extubated on the very next day after chest closure, during which patient maintained good hemodynamics. Patient was discharged on the 8th postoperative day. Post-operative echo was suggestive of normal functioning mitral valve prosthesis. On the 7th postop day a repeat cardiac CT was done (Fig. 3).

\section{Discussion}

Glenner and Grimley, ${ }^{1}$ in their classic description, divided the extra adrenal portion of the paraganglionic system into four groups on the basis of location, innervation, and histology: branchiomeric, intravagal, aortic sympathetic, and visceral-autonomic. Paraganglion are minute 

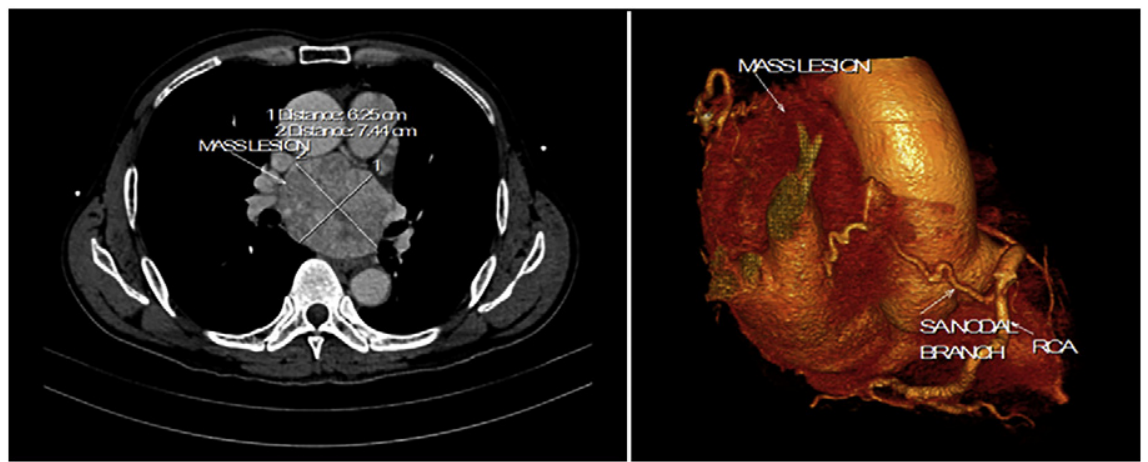

Fig. 1. Location of paraganglioma in mediastinum relation to aorto pulmonary window.

macroscopic and microscopic cell groups associated with the autonomic nervous system. All paraganglia, including chemodectoma, store catecholamine and other neurotransmitter substances. Tumors of the extra adrenal paraganglionic system, paraganglioma, may or may not secrete noradrenaline in their clinical presentation. Although there are some cases in this study of secreting tumors, these tumors should not be called pheochrornocytorna; this term is reserved for secreting tumors of the adrenal gland. ${ }^{1}$ Thoracic paragangliomas are located mainly in the posterior and middle mediastinum. The former arise from the region of the aortosympathetic or intravagal paraganglia, and the latter arise around either the branchial arch structures (i.e., coronary and aortic pulmonary paraganglioma) or the visceral- autonomic paraganglia of the cardiac atria and interatrial septum. ${ }^{2}$

Paraganglioma are highly vascular tumours and around half of them are found incidentally. Most of the Symptoms are due to catecholamine hyper secretion of the tumours. ${ }^{3}$ Its proximity to great vessels makes it difficult to excise. Surgical excision is the only accepted treatment. Excision should always be planned on a cardiopulmonary bypass. Paragangliomas are closely related to pheochromocytomas and are similar histologically. Sympathetic paragangliomas usually secrete catecholamine and are located in the sympathetic paravertebral ganglia of thorax, abdomen, and pelvis. In contrast, most parasympathetic paragangliomas are non-functional and located along the glossopharyngeal and vagal nerves in the neck and at the base of the skull. Catecholamine-secreting paragangliomas often present clinically like pheochromocytomas with hypertension, episodic headache, sweating, and tachycardia. However, the distinction between pheochromocytoma and paraganglioma is an important one because of implications for associated neoplasms, risk for malignancy, and genetic testing.

Chest tomography, computed tomography, magnetic resonance imaging, angiography, selective venous sampling, and two-dimensional echocardiography are the most frequently used investigations in
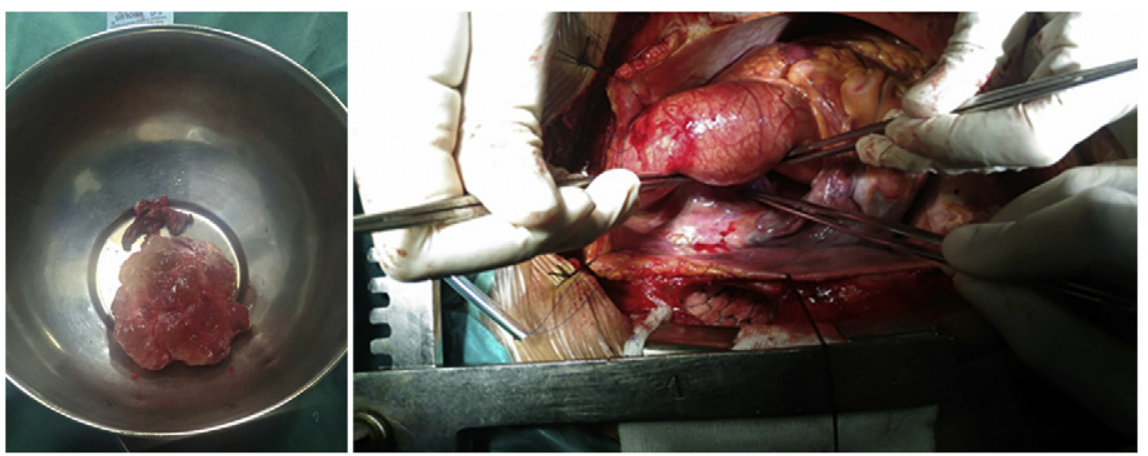

Fig. 2. Resected mass, and its relation to aorto pulmonary window Intraoperative mass.
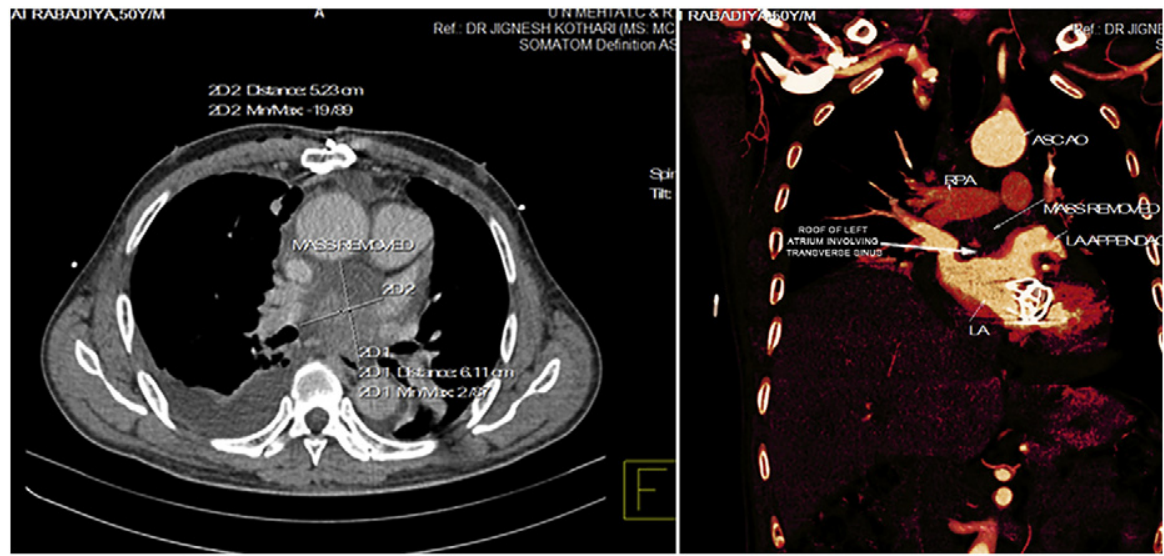

Fig. 3. The post-operative CT scan after resection. 
diagnosing intra thoracic paragangliomas. ${ }^{4,5,6,7}$ Incidence rate of intra thoracic paraganglioma is $<2 \%$ of the functioning paragangliomas. Total surgical resection of the mediastinal paraganglioma is the best treatment at present. We strongly recommend a median sternotomy to gain access to all structures of the anterior and middle mediastinum. Cardiopulmonary bypass should be used, if necessary, to mobilize the great vessels, control the bleeding, and provide exposure for complete resection.

In our patient the main symptom was dyspnea and the patient was preoperatively hypertensive. We accidently came to know about the mass on doing coronary angiography, so we further planned for CT scan in which we could confirm the location of the mass. We operated the patient and did mitral valve replacement with mechanical valve of size $27 \mathrm{~mm}$ and removed the mediastinal mass. Postoperatively patient was completely asymptomatic. Our case is very rare where mediastinal mass excised with mitral valve replacement. To our best of knowledge till now such case is rarely reported.

\section{Funding}

This work was supported by U.N. Mehta Institute of Cardiology and Research Center itself and received no specific grant from any funding agency, commercial or not-for-profit sectors.

\section{Conflict of interest}

None declared.

\section{References}

1. Glenner GG, Grimley PM. Tumors of the extra-adrenal paraganglion system (including chemoreceptors). Atlas of Tumor Pathology, Second Series, Fascicle 9. Washington, DC: Armed Forces Institute of Pathology; 1974. p. 68-69.

2. Cohen PS, Israel MA. Biology and treatment of thoracic tumors of neural crest origin. In: Roth JA, Ruckdeschel JC, Weisenburger TH, editors. Thoracic oncology. Philadelphia: Sanders; 1989. p. 520-540.

3. Wald O, Shapira OM, Murar A, Izhar U. Paraganglioma of the mediastinum: challenges in diagnosis and surgical management. Journal of cardiothoracic surgery 2010 Mar 31;5(1):19.

4. Dunn GD, Brown MJ, Sapsford RN, et al. Functioning middle mediastinal paraganglioma (phaeochromocytoma) associated with intercarotid paragangliomas. Lancet 1986;327(8489):1061-1064.

5. Cueto-Garcia L, Shub C, Sheps SG, Puga FJ. Two dimensional echocardiographic detection of mediastinal pheochromocytoma. Chest 1985;87(6):834-836.

6. Spizarny DL, Rebner M, Gross BH. CT evaluation of enhancing mediastinal masses. $J$ Comput Assist Tomogr 1987;11(6):990-993.

7. Barakos JA, Brown JJ, Brescia RJ, Higgins CB. High signal intensity lesions of the chest in MR imaging. J Comput Assist Tomogr 1989;13:797-802. 